Revue française de la traduction

\title{
Particularités de la traduction du texte de presse : le problème du titre journalistique
}

Étude de cas

\section{Carmen-Ecaterina Aştirbei}

\section{(2) OpenEdition}

Journals

Édition électronique

URL : http://journals.openedition.org/traduire/85

DOI : $10.4000 /$ traduire.85

ISSN : 2272-9992

Éditeur

Société française des traducteurs

Édition imprimée

Date de publication : 15 décembre 2011

Pagination : 33-48

ISBN : 039-773X

ISSN : 0395-773X

Référence électronique

Carmen-Ecaterina Aștirbei, «Particularités de la traduction du texte de presse : le problème du titre journalistique », Traduire [En ligne], 225 | 2011, mis en ligne le 10 février 2014, consulté le 19 avril 2019. URL : http://journals.openedition.org/traduire/85 ; DOI : 10.4000/traduire.85 


\section{Particularités de la traduction du texte de presse : le problème du titre journalistique. Étude de cas(1)}

Carmen-Ecaterina Aştirbei

\section{Caractéristiques du texte journalistique}

\subsection{Repères généraux d'analyse}

Le texte de presse est peut-être l'un des domaines les moins discutés du point de vue traductologique. Le vrai problème, de nos jours, est le fait qu'il n'y a pas de traduction journalistique proprement dite, mais des adaptations au contexte social, économique ou culturel de la langue cible. La traduction journalistique respectera, en général, la lettre du texte d'origine. D'où la confusion qui règne parmi les spécialistes en traductologie.

Pour bien traduire ou adapter le texte de presse, il faut que le traducteur soit conscient des caractéristiques d'un tel type de texte. Le texte journalistique n'est pas construit au hasard : plus que tout autre texte, il est soumis à des contraintes spécifiques. Le traducteur doit être, en principe, journaliste, ou bien maîtriser les règles de rédaction du texte de presse.

Les traducteurs sont confrontés principalement à deux sortes de textes à caractère journalistique : les communiqués de presse et les articles. En ce qui concerne les communiqués de presse, leur traduction assure une diffusion plus large des messages qu'ils véhiculent. Le traducteur doit savoir aussi que les organisations internationales publient d'habitude les communiqués dans leurs langues officielles. Les communiqués de presse comportent des informations plus précises et d'un degré d'importance plus élevé, puisqu'ils contiennent des faits très récents et d'actualité, susceptibles de concerner tout le monde.

Quant aux articles à traduire, il y a d'une part ceux que font traduire dans une ou plusieurs langues des revues destinées à une région particulière, (par exemple, la National Geographic Society publie une édition française de sa revue). D'autre part, certaines publications paraissent simultanément dans plusieurs langues (par exemple, le Forum du désarmement est une revue

(1) La documentation et la recherche en vue de la publication de cette étude se sont déroulées dans le cadre du programme POSDRU/88/1.5/S/47646, cofinancé par le Fond Social Européen, par l'intermédiaire du Programme Opérationnel Sectoriel Développement des Ressources Humaines 2007-2013. 
publiée en français et en anglais par l'Institut des Nations Unies pour la recherche sur le désarmement). Une troisième catégorie est constituée par les articles traduits quotidiennement, d'une langue source dans une langue cible, afin d'offrir au public des informations d'actualité, d'ordre général ou appartenant à divers domaines d'activité. Par exemple, certains articles parus dans Le Monde peuvent être traduits dans des quotidiens roumains (Adevărul, Evenimentul zilei) afin d'apporter dans la langue cible le même contenu informationnel.

Le problème, du point de vue traductologique, est que rares sont ceux qui sont spécialisés dans la traduction de tels textes, laquelle exige à la fois des compétences de traducteur et un talent journalistique particulier. Le cas du traducteur-journaliste étant assez rare, celui qui traduit un texte relevant du journalisme est tenu de connaître et, dans la mesure du possible, d'appliquer les règles qui s'imposent aux journalistes. Un cas fréquent est l'adaptation du texte original, si l'auteur de celui-ci a enfreint les règles, ce qui est souvent le cas.

En effet, un texte de presse est un texte d'actualité qui contient des nouvelles. Une nouvelle est un fait récent, présenté dans son contexte et de nature à intéresser le lecteur. Le but est de conserver la fraîcheur de la nouvelle ; à cette fin, le traducteur doit connaître le contexte de la nouvelle ou se documenter à son sujet. Mais, surtout, sa tâche essentielle sera de susciter et maintenir l'intérêt du lecteur en employant des techniques spécifiques.

La clarté est la condition essentielle du texte de presse. Tout texte journalistique doit être intelligible et attrayant. Ce qui est obscur ou maladroit doit être proscrit. La forme, elle aussi, doit être prise en considération : le traducteur a la mission de présenter des informations particulièrement arides dans un style explicite et vivant (ce qu'on appelle en traductologie adaptation au contexte de la langue cible).

Pour ce faire, il faut impérativement tenir compte des destinataires du texte. Si l'information est destinée à un public averti, il faut employer un style approprié et la terminologie correcte. L'exactitude terminologique contribue en effet à conférer de l'autorité aux informations qui sont présentées.

Mais il arrive que les destinataires du texte soient des profanes, des personnes peu familiarisées avec le domaine en question. Par exemple, un communiqué de presse est généralement lu par un journaliste qui n'a qu'une connaissance approximative du sujet présenté. D'habitude, les informations sont destinées au grand public et doivent donc être assimilées par le plus grand nombre de lecteurs possible. La clarté et la simplicité du style sont de rigueur. Par exemple, dans un discours de vulgarisation, il convient de substituer la structure "le virus du sida " au "virus de l'immunodéficience humaine " et à l'abréviation "VIH ". De même, il est préférable d'écrire " organes producteurs de sang " plutôt qu'" organes hématopoïétiques ". Le discours de presse est un discours de vulgarisation, adressé au grand public, raison pour laquelle les termes trop spécifiques doivent être évités. A contrario, le traducteur doit, lors de sa traduction, respecter la lettre et la terminologie du texte source si cela est nécessaire. Un article 
scientifique, même si c'est de la presse écrite, sera traduit d'une manière appropriée ; toute adaptation va altérer le contenu informationnel d'origine. Du point de vue de la théorie du skopos (2) aussi, formulée par Katarina Reiss, un texte à visée informative sera traduit en respectant l'invariance du contenu et en mettant, lors de la traduction, l'accent sur le texte cible et sur sa fonction dans la langue et la culture d'origine.

Tout énoncé qui manque de clarté est de nature à dérouter le lecteur. Le traducteur a le devoir de se documenter ou de consulter l'auteur pour éclaircir les points confus. La clarté passe le plus souvent par la simplicité. Par exemple, une traduction qui conserve des abréviations sera obscure pour le lecteur du texte cible. Un titre tel que " $\mathrm{La} \mathrm{CCl}$ organise le premier forum des entreprises " traduit en roumain par " $\mathrm{CCl}$ organizează primul forum al întreprinderilor " sera obscur et même incompréhensible. Le traducteur se doit d'expliciter le sigle $(\mathrm{CCl}=$ Chambre de Commerce Internationale), afin de rendre le titre compréhensible pour le public roumain.

Un autre problème est constitué par le nom des personnes citées qui doit être suivi de leur titre, fonction ou qualité ; ce qui compte, cependant, ce n'est pas l'exactitude du titre sur le plan administratif, mais le recours à une dénomination qui permette de situer la personne à l'aide de sa fonction. De même, s'il est question d'une personne en début d'article, le lecteur ne se souviendra pas de son nom trente lignes plus loin. Dans un tel cas, le traducteur devra répéter la fonction de cette personne.

Pourtant, le désir d'être clair ne mènera pas le traducteur à la simplification abusive ; il ne faut pas prendre le lecteur pour un simple d'esprit. Le traducteur du texte de presse doit se situer en deçà du niveau de compréhension des lecteurs les plus cultivés, le but étant d'élever le niveau de culture du public ordinaire.

En traduction, il est parfois difficile d'être clair et concis, et de restituer l'information de façon exacte et complète, surtout quand celle-ci est assez technique ou quand le traducteur doit recourir à des explicitations. II convient alors de faire un arbitrage entre les différentes méthodes de traduction.

\subsection{Caractéristiques du titre journalistique}

La traduction des titres journalistiques suppose premièrement un rappel des caractéristiques du titre, élément décisif pour éveiller l'intérêt d'un lecteur qui, de prime abord, n'est pas orienté. Ce type de traduction permet au traducteur de donner toute sa mesure, car il dispose là d'une grande liberté de manœuvre.

(2) Le terme skopos, d'origine grecque, signifie but ou objectif et a été introduit pendant les années 1970 par le théoricien allemand Hans J. Vermeer comme un terme technique désignant le but du texte d'arrivée et de l'action traduisante. 
Dans le monde journalistique, un titre est à la fois court et explicite. Par exemple, pour introduire un bref portrait d'un arbitre féminin de football, le journal Le Monde publie le titre "Madame l'arbitre ". Afin d'être concis, un moyen commode consiste à indiquer brièvement le contexte et à présenter ensuite l'information (exemple classique de titre journalistique : " Jolo : Paris craint pour ses deux otages " - Le Figaro)(3).

Dans la traduction il faut pourtant expliciter un titre trop laconique et ne pas se livrer à des contorsions extraordinaires à la seule fin d'être lapidaire ou de transposer le texte dans un style journalistique concentré.

De même, il faut tenir compte du style privilégié par chaque journal : Libération utilise des titres percutants, tandis que Le Monde a souvent recours à des titres sobres mais longs. De même, le traducteur devra chercher la tonalité qui convient à son public cible.

En effet, le titre doit accrocher sans racoler. Un titre trop accrocheur peut nuire à l'impression de sérieux que l'on veut donner aux informations diffusées. Le titre choc est préféré par la presse populaire, tandis que les journaux de haute tenue le réservent aux informations plus frivoles.

L'exemple classique donné dans toutes les études de traductologie est le cas d'un tueur en série qui terrorisait New York. Quand il a été enfin arrêté, le New York Post a titré en caractères gras un seul titre : "CAUGHT". La psychose de la ville a cessé et les habitants ont compris aussitôt que le tueur avait été appréhendé. Pourtant, la traduction de ce titre aurait dû être explicitée dans toute autre langue d'arrivée, puisque le public cible ne connaissait pas le contexte dans lequel l'article avait été écrit.

La recherche d'un titre choc peut conduire aux pires échecs, en traduction aussi. Si le titre doit attirer l'attention, son principal attrait doit provenir de l'intérêt qu'il peut susciter auprès du public cible. Chaque titre a un intérêt intrinsèque au regard de la nouvelle qu'il résume. Par exemple, un titre comme : "Un nouveau médicament contre le sida va être commercialisé au sein de l'Union européenne " (Le Monde) peut intéresser tous les publics. Mais un titre plus ronflant que celui déjà cité est préféré, parce que le lecteur va se rendre compte, à la lecture de l'article, que les vertus du médicament en question sont bien limitées.

II y a aussi plusieurs manières de rédiger les titres dans des langues différentes. Par exemple, le langage des titres n'est pas le même dans le monde anglo-saxon qu'en France. Dans le monde anglophone on utilise avec prédilection certains mots courts pour des raisons d'économie linguistique.

(3) René Meertens, La traduction des textes journalistiques, http://www.foreignword.com, consulté le 10 décembre 2010. 
Le traducteur doit s'efforcer de trouver un titre personnel, sans rester trop fidèle à l'original. II y a plusieurs méthodes de traduction qui lui permettent d'utiliser le titre pour donner un peu plus de vitalité à un article ou à un communiqué de presse, anodin de prime abord. Parmi ces méthodes traductologiques nous pouvons énumérer(4) :

1) l'allusion et le jeu de mots : "La voiture électrique démarre " (Le Monde); "Un électrochoc pour Moulinex " (Le Monde) ; "Les machines à laver, c'est le propre de l'art " (Le Figaro);

2) la métaphore : "La pêche aux amendes au large de Guernesey " (Le Monde);

3) le paradoxe : "Le patron est à la CGT et les ouvriers sont des capitalistes " (Le Monde);

4) l'effet de surprise : "Radko Mladic, ses abeilles et ses colonels " (Le Monde).

Le traducteur doit conserver le même procédé de construction du titre ou bien, si cela n'est pas possible, il doit recourir à une méthode de compensation (selon le concept d'équivalence dynamique avancé par Nida(5)).

Il y a des cas où la recherche de l'originalité à tout prix peut être exagérée. Des titres comme "Le plan-plan Bayrou pour les universités " ou bien "Les ovins atteints de 'tremblante' quittent l'assiette " (in René Meertens, op. cit.) peuvent intriguer le lecteur qui va abandonner la lecture de l'article une fois qu'il aura compris l'allusion. De l'autre côté, le travail du traducteur est difficile : il doit rendre dans la langue cible l'allusion faite par l'auteur de l'article. Une « équivalence dynamique " sera, dans ce cas, préférable.

\section{Traduction des titres de presse}

Les titres de presse sont très marqués par le genre discursif et par la tradition journalistique ; d'habitude, les titres constituent des segments courts qui ont de multiples fonctions et qui, parfois, exigent que le traducteur ait recours à une reconstruction complexe du sens. Dans son article, "La traduction français-espagnol des titres journalistiques du Monde diplomatique, un exemple de tension entre adéquation et acceptabilité "(6), Gemma Anjúdar Moreno fait une analyse de traduction journalistique appliquée à un corpus de titres du mensuel français $L e$ Monde diplomatique et de leurs versions espagnoles. Dans ce qui suit, nous allons analyser ces titres de presse du point de vue de la " tension " entre adéquation et acceptabilité, utiliser les exemples de la langue source et observer par quel procédé se réalise la traduction en langue cible qui est, dans notre étude de cas, le roumain. Le vrai problème semble, en effet, être les implications de la culture de départ qui orientent la démarche globale des traducteurs et le choix des méthodes face aux titres à traduire.

\footnotetext{
(4) Ibid.

(5) Eugene Nida, The Theory and Practice of Translation, Brill Academic Publishers, 2003, p. 1.

(6) Gemma Anjúdar Moreno, un exemple de tension entre adéquation et acceptabilité, http://www.translationdirectory.com/article1135.htm, consulté le 10 décembre 2010.
} 


\subsection{Préparation de la démarche traductologique}

En ce qui concerne la spécificité des titres de presse, on admet aujourd'hui que le traducteur doit les adapter à la situation de communication spécifique dans laquelle vont s'inscrire ces textes. Afin de choisir la méthode de traduction, le traducteur doit tenir compte, parmi d'autres facteurs, du genre discursif du texte de départ, de l'auteur de l'article, du lieu et du moment de production, mais aussi du public cible et de la culture à laquelle il appartient. En effet, il s'agit ici d'un " acte de médiation linguistique et culturelle "(7) qui a des implications considérables dans la traduction du discours journalistique. Aux problèmes de nature culturelle, qui sont très épineux, s'ajoutent les particularités des genres de la presse écrite et les traditions journalistiques dans chaque culture.

Un des enjeux qui caractérisent la complexité du texte de presse est le titre. La condition essentielle est que le titre soit concis, bref, voire choquant dans certains cas et pour certains genres journalistiques. Les titres sont des segments courts, mais qui fonctionnent du point de vue sémantique comme une entité complète ; du point de vue traductologique, ils présentent un intérêt particulier grâce à leurs fonctions multiples et à leur valeur cataphorique accentuée. Le traducteur va saisir les implications du titre de presse et donner, en langue cible, une construction de sens complexe et rétroactive.

Le corpus de titres que nous allons analyser nous permettra de mettre en évidence les procédés de traduction employés lors de la traduction des titres de presse tout comme les priorités qui régissent le processus de traduction choisies en fonction des divergences de traduction. Nous allons d'abord essayer de voir quel est le degré d'intervention du traducteur dans la question du titre de presse traduit, et ensuite préciser quelles sont les conséquences des stratégies de traduction appliquées aux textes dans la langue d'arrivée.

\subsection{Exemples de titres traduits extraits du Monde diplomatique}

Les titres que nous allons choisir dans ce qui suit ont parus dans le mensuel français Le Monde diplomatique, mensuel qui jouit d'un grand prestige par son influence et par sa diffusion internationale.

Pour ne pas mélanger les genres, nous allons fonder notre analyse traductologique exclusivement sur un genre de la presse écrite : l'article journalistique. Des genres tels que l'éditorial ou le reportage pourraient mener notre réflexion vers d'autres paramètres. En général, lorsqu'il s'agit du mensuel Le Monde diplomatique, on parle d'articles très marqués du point de vue idéologique et qui abordent des questions sociales, politiques et économiques. La structure interne du texte se fonde sur des séquences argumentatives qui se cristallisent de différentes

(7) Ibid. 
manières. Le discours journalistique est fondé sur des stratégies argumentatives complexes ; il emploie aussi un éventail de connecteurs discursifs, des mécanismes empathiques, des exemples ou des citations authentiques pour soutenir ou réfuter des arguments.

De plus, le traducteur doit tenir compte du fait que l'intérêt de ces documents réside aussi dans leur condition de textes réunissant des marques visuelles (images, typographie, caractères employés, segmentation du texte) et des structures d'ordre discursif fondées sur le type explicatif et argumentatif, autant d'éléments qui ne sont pas toujours transposables en langue cible (dans notre cas, en roumain).

Les titres constituent une catégorie à part, qui peuvent même être compris en tant qu'unités de traduction. C'est en fonction du titre traduit que l'on peut faire une comparaison entre le texte de départ et le texte d'arrivée (Toury, 1995 ; Nord, 1998, in Gemma Anjúdar Moreno, op. cit.). Christiane Nord considère aussi que "les titres constituent des segments textuels complexes, à cause de leur polyfonctionnalité à plusieurs niveaux de l'organisation discursive " (ibid.). Selon Christiane Nord, citée par Gemma Anjúdar Moreno (op. cit.), les différentes fonctions auxquelles recourent les titres peuvent se rapporter aux fonctions de base du langage :

- la fonction phatique (ouvrir la communication entre l'auteur et le lecteur de l'article) ;

- la fonction appellative (séduire le lecteur pour l'amener à lire l'article) ;

- la fonction informative (annoncer le sujet qui va être développé dans l'article) ;

- la fonction expressive (véhiculer des attitudes ou des émotions de l'auteur à l'égard des événements du monde ; d'une façon plus ou moins marquée, en fonction de l'article et de la tradition journalistique).

Quant aux titres du Monde diplomatique, ils ont une structure spécifique : ils présentent des faits en synthétisant la thèse qui va être défendue par l'auteur, parfois d'une manière directe et suggestive :

(1) Où va le Maroc? (juillet - août 1999);

Marocul, încotro ? (nous avons recours à une modulation pour que la phrase ne soit pas forcée en roumain) ;

(2) Se battre, se rebeller ou se désintégrer (janvier 2003) ;

Să lupţi, să te revolţi sau să te dezintegrezi (nous avons personnalisé et rendu le titre d'origine plus familier en langue cible) ;

(3) Le couple nippon - américain à l'heure du soupçon (avril 1999);

Parteneriatul japonezo - american la ceas de îndoială (nous avons choisi d'expliciter le contexte par une métaphore permettant d'ajouter plus de cohérence au titre traduit en roumain). 
Comme on peut le constater, les titres fonctionnent comme une entité complète du point de vue sémantique et polyfonctionnelle dans la culture de départ, mais aussi dans la culture d'arrivée. Ces caractéristiques font de leur interprétation immédiate et de leur traduction ultérieure un processus très riche qui sera analysé dans ce qui suit.

\subsection{Analyse contrastive des titres traduits}

Par une analyse contrastive on peut décrire, du point de vue traductologique, les mécanismes récurrents utilisés par les traducteurs pour rendre le sens du titre de la langue de départ avec le matériau linguistique et discursif de la langue d'arrivée. En effet, ces mécanismes, appelés aussi " stratégies de traduction ", relèvent du choix personnel du traducteur et rendent compte également de son interprétation du texte source. Décrire les stratégies de traduction appliquées nous permettrait de souligner la stratégie de traduction générale pouvant orienter la démarche globale des traducteurs dans le problème des titres à traduire.

Dans son article, Gemma Anjúdar Moreno (op. cit.) remarque tout de même que la fréquence d'apparition des stratégies de traduction, qui peuvent être "dominantes " ou " secondaires ", a un rôle important dans le processus de traduction des titres journalistiques. La même auteure souligne le fait que la régularité des tendances dans la traduction est un très bon indicateur pour le choix général des traducteurs en matière de titres traduits : " les choix de traduction observés dans le corpus peuvent s'inscrire dans une échelle qui oscille entre les solutions qui reflètent le texte de départ d'une façon littérale ou presque et d'autres qui n'ont pas de rapport de conjonction totale avec le texte de départ (modulations, modifications et mutations selon le degré d'éloignement sémantique) "(8).

Dans ce qui suit, nous allons reproduire le schéma proposé par Gemma Anjúdar Moreno (op. cit.) dans l'article cité et qui résume les stratégies de traduction établies :

- Intervention du traducteur :

I. traductions littérales et quasi littérales (dominantes) ;

II. traductions non littérales (secondaires)

- modulations ;

- modifications ;

- additions et guillemets ;

- changement d'un acte de parole ;

- transformation de la structure bimembre du titre de départ ;

- mutations : changement radical de sens ;

(8) K. van Leuven - Zwart, Translation and Original: Similarities and Dissimilarities, Target 1: 2, John Benjamins, Amsterdam, 1989, p. 156. 


\subsubsection{Traductions littérales et presque littérales}

Les traductions littérales des titres français sont très fréquentes, comme on l'a déjà observé. Citons quelques exemples:

(1) Double piège pour l'Afghanistan (février 2009) ;

Capcană dublă pentru Afganistan ;

(2) Quand les pauvres séduisent les banques (avril 1999) ;

Când săracii seduc băncile (aprilie 1999).

Dans ces exemples, on peut observer qu'il n'y a pas de divergence entre le segment de départ et le segment d'arrivée, parce que la traduction est fidèle au texte de départ.

II y a aussi d'autres traductions presque littérales où un élément du titre français subit une très petite modification, mais sans produire un changement de sens global ; ainsi, dans certains titres, un terme au singulier peut-il être traduit par un terme au pluriel :

(3) La vague du chômage déferle sur la Chine (janvier 1999) ;

Valurile şomajului inundă China (nous avons recouru ici à un verbe plus expressif en langue cible (" a inunda ") pour que le titre ne semble pas maladroit au lecteur roumain) ;

(4) Histoire secrète des négociations de Rambouillet (mai 1999) ;

Istoria secretă a negocierilor de la Rambouillet (le traducteur a ajouté ici une détermination absente du texte de départ).

Les traductions littérales et presque littérales constituent la modalité la plus fréquente dans le corpus analysé. Du point de vue de l'intervention du traducteur, Gemma Anjúdar Moreno considère que ce type de traduction se situe à un niveau secondaire. Elle montre que le traducteur a jugé qu'il pouvait respecter les particularités structurelles et formelles du texte d'origine.

Notre conclusion est donc que, dans un grand nombre de cas, même si les titres journalistiques appartiennent à des genres discursifs divers, ils sont traduits littéralement. Les modifications qui se produisent en traduction sont, ainsi, mineures, et la plupart du temps, imposées par les exigences de la nouvelle situation de communication. Cette fidélité envers le titre traduit provient en règle générale du respect par l'auteur de l'article original, mais aussi du fait que, dans notre cas, les normes de construction des titres journalistiques en français ressemblent aux normes de la langue cible (ici, le roumain). Le fait que la traduction littérale soit la stratégie la plus utilisée suggère que, lors de la traduction, le principe d'adéquation est prioritaire : les traducteurs ont choisi d'importer dans le texte traduit les éléments linguistiques et culturels propres au texte source. Pourtant, les exemples les plus intéressants sont ceux où les traducteurs choisissent volontairement de s'écarter du texte source, sans y être contraints par les exigences linguistiques du texte d'origine. Dans ce qui suit, nous allons analyser certains cas où la "désobéissance " du traducteur produit des versions inouies de traduction. 


\subsubsection{Traductions non littérales}

\subsubsection{Modulations}

Les modulations apparaissent " lorsque le choix de traduction a un rapport hyperonymique avec le texte de départ : la traduction développe un aspect syntaxique, sémantique ou stylistique de disjonction (plus général) tout en gardant certains liens de convergence "(9). Dans le cas des titres de presse, presque toutes les modulations sont de type sémantique, comme l'illustrent les exemples suivants :

(1) Controverses passionnées à propos des sectes (mai 1999);

Controverse arzânde cu privire la subiectul sectelor (l'adjectif " passionnées " est traduit en langue cible par un gérondif, "arzânde ", solution inédite. L'emploi de l'adjectif " arzătoare " serait possible, lui aussi) ;

(2) Les pays du Sud rongés par les pesticides (avril 1999);

Ţările din sud, afectate de pesticide

Ţările din sud, victime ale pesticidelor (les deux solutions sont possibles ; on observe la transformation, en traduction, de l'adjectif verbal "rongés par " en adjectif qualificatif (" afectate de ", "victime ale ", solution plus appropriée en langue cible) ;

Dans les deux cas exposés, les choix dénominatifs du traducteur sont évidemment plus généraux du point de vue sémantique que leurs correspondants français. Cette stratégie de traduction se caractérise par une perte de précision et d'intensité sémantique qui réduit la charge émotionnelle des titres traduits en roumain.

\subsubsection{Modifications}

Les modifications apparaissent lorsque la solution de traduction se place dans un rapport contrastif avec le texte de départ, mais justifié par une interprétation plus profonde du titre journalistique. Dans son article, Gemma Anjúdar Moreno établit les catégories suivantes de modifications :

- ajout de guillemets ;

- changement d'un acte de parole ;

- suppression d'un élément locatif ;

- transformation de la structure bimembre du titre de départ.

Dans ce qui suit, nous allons analyser ces stratégies du point de vue de leur emploi dans la traduction vers la langue cible (vers le roumain, dans notre cas). En effet, ces méthodes traductologiques ont toutes le même but : " normaliser ", " adapter " le titre journalistique de départ pour obtenir un titre journalistique plus naturel et adapté aux lecteurs roumains.

(9) Gemma Anjúdar Moreno, op. cit., p. 23. 


\subsubsection{Addition de guillemets}

Dans la traduction des titres de presse, le traducteur peut prendre des libertés et incorporer un terme ou une expression entre guillemets, même si elles ne sont pas présentes dans le texte de départ. En voici un exemple :

(1) Amère victoire pour les mineurs roumains (février 1999);

"Victorie " amară pentru minerii români (dans ce cas, on souligne grâce aux guillemets l'ironie exprimée dans le titre de départ ; le choix de l'emploi des guillemets est facultatif, mais, en les utilisant, on ajoute davantage de cohérence au titre dans la langue cible).

Comme on peut l'observer, dans la traduction vers le roumain, la fonction principale de ce signe de ponctuation est d'expliciter une distance énonciative par rapport au terme entre guillemets. L'addition des guillemets dans la traduction implique, entre autres, un doute jeté sur la justesse ou la précision du terme (les guillemets fonctionnent ici, en effet, comme renforcement de l'adjectif " amère " qui qualifie " la victoire "). Dans ce cas il s'agit donc d'une interprétation corrective de la part du traducteur, qui indique l'apparition d'une nouvelle voix ou d'un nouveau point de vue.

Dans d'autres exemples, la fonction des guillemets ajoutés à la traduction est de marquer l'introduction d'un choix créatif ou surprenant, propre au caractère ludique de la presse française :

(2) Journalistes à tout faire de la presse américaine (février 1999) ;

Jurnalişti " gata de orice " în presa americană

Dans ce cas-ci, le signe de ponctuation marque dans la traduction une expression surprenante ou peu habituelle pour les lecteurs roumains (en effet, l'expression " journalistes à tout faire " est issue de l'expression " bonne à tout faire "). II s'agirait donc d'une sorte de " commentaire " textuel de la traduction.

\subsubsection{Changement d'un acte de parole}

Si l'on analyse la traduction des titres de presse du Monde diplomatique, on observe quelques occurrences où l'acte de parole du segment de départ, une exclamation ou une interrogation rhétorique, est rendu en roumain par une assertion. Cette intervention de la part du traducteur implique, en tout cas, une perte de force expressive du texte de départ, comme dans les exemples suivants :

(1) Danger, prolifération étatique! (février 1999);

Pericolul proliferării statale ou bien :

Atenţie, proliferare statală ! (on a changé la nature de la phrase, on l'a traduite dans une tonalité plus neutre et on a même renoncé au point d'exclamation) ; 
(2) L'alliance du Mecklembourg fera-t-elle école? (mars 1999) ;

Alianţa Mecklembourg ca exemplu

Le premier titre contient une exclamation, tandis que le deuxième est fondé sur une interrogation, stratégie qui vise à impliquer le lecteur français. Dans ces exemples, le lecteur va se trouver émotionnellement impliqué dans le contenu de l'énoncé. En revanche, les titres traduits en roumain sont moins marqués du point de vue énonciatif, moins emphatiques et donc moins proches du lecteur.

Mais il y a des cas où à la perte de force énonciative dans la traduction s'ajoute un éloignement sémantique plus grand entre le texte source et le texte cible. Ainsi, par exemple, le titre suivant et sa traduction :

(3) La Belgique survivra-t-elle aux élections de 1999 ? (juin 1999)

Sistemul belgian în criză generală

Nous pouvons ajouter aussi une variante littérale de traduction de ce titre de presse : Belgia va supravieţui oare alegerilor din 1999 ? Pourtant, la première variante nous semble plus adaptée au public cible. II y a donc ici un éventail de possibilités interprétatives ouvert par l'interrogation qui devient une assertion orientée vers la négativité absolue : " criză generală ". Cette traduction implique une vraie prise de position de la part du traducteur, choix assez risqué d'ailleurs. Or, il est évident que le traducteur a reconstruit le titre à partir de paramètres différents, probablement à partir de la lecture globale du texte entier.

\subsubsection{Transformation de la structure bimembre du titre de départ}

II y a des cas où la stratégie de traduction appliquée implique la transformation d'un titre bimembre en français en titre unimembre en roumain. Voici quelques exemples de cette modification d'ordre syntaxique et pragmatique :

(1) Libre-échange, la dernière Bastille (janvier 1999);

\section{Liberul schimb, ultima redută}

Cet exemple illustre l'importance de la prise en compte du genre discursif pour la traduction des titres de presse. C'est le genre discursif qui pèse sur les décisions du traducteur visant à l'acceptabilité de la traduction dans la culture d'arrivée.

L'exemple ci-dessus est un modèle de titre bimembre, modalité très habituelle en français pour les articles journalistiques de type argumentatif, tandis qu'en roumain ils sont moins utilisés. Gemma Anjúdar Moreno souligne le fait que la suppression d'un élément locatif du texte source est une stratégie qui permet au traducteur d'obtenir un titre unimembre en roumain. La fonction de l'élément locatif est d'aider le lecteur à situer l'article dans le cadre géographique mondial sans avoir besoin de lire l'article en entier. Pour illustrer ce fait, nous allons donner deux exemples: 
(2) À Berlin, face à face des intellectuels de l'Est et de l'Ouest (mars 1999)

Faţă în faţă, la Berlin, confruntare între intelectualii din est şi din vest

(3) En Turquie, procès du peuple kurde

Proces al poporului kurd în Turcia

On observe que la structure bimembre du titre français, structure classique d'ailleurs, ne peut pas être respectée dans la traduction. Placé toujours en position initiale dans la structure du titre français, cet élément locatif fonctionne comme un " marqueur d'univers de discours "(10), car il ouvre la voie à un espace sémantique dans lequel le lecteur établit son interprétation. Si le traducteur renonce à la structure binominale, cela signifie qu'on élimine du titre roumain un élément qui pourrait faciliter la localisation spatiale de l'article. En outre, cette intervention du traducteur peut être mise en rapport avec les traditions journalistiques de chaque culture : supprimer l'élément locatif, c'est une stratégie de traduction très simple pour obtenir un titre unimembre, plus naturel en roumain.

\subsubsection{Mutations}

Selon Gemma Anjúdar Moreno, cette catégorie correspond aux glissements de sens qui éloignent la traduction du texte de départ à tel point que le rapport d'équivalence est atténué. La fréquence d'apparition de cette méthode de traduction est nettement inférieure à celle des catégories dont nous avons déjà débattu.

Cette méthode traductologique se situe, selon Gemma Anjúdar Moreno (op. cit.), au niveau supérieur d'intervention du traducteur et a pour rôle d'adapter les versions en langue cible aux nouvelles conditions de la culture d'arrivée. Dans de nombreux cas, il est même possible de parler d'adaptations plus que de traductions, à cause des écarts de sens qui sont difficiles à expliquer.

Dans son article, Gemma Anjúdar Moreno classe les mutations en deux grands groupes qui répondent à la même logique : la volonté de "normaliser " le titre français et de le rendre plus acceptable pour la culture d'arrivée. Dans le premier groupe, la stratégie de traduction consiste à remplacer un titre subjectif par un titre plus objectif, qui résume le contenu de l'article d'une façon beaucoup moins affective (Gómez Montpart, 1982)(11). Les titres suivants, donnés comme exemple par Gemma Anjúdar Moreno, illustrent ce choix de traduction :

(1) - "On ne se prostitue pas par plaisir " (février 2003)

"Cauzele economice şi sociale ale prostitutiei "

(10) J.-M. Adam, Éléments de linguistique textuelle. Théorie et pratique de l'analyse textuelle, Liège, Mardaga, 1990.

(11) In Gemma Anjúdar Moreno, op. cit. 
(2) - "En Iran, Islam contre Islam " (juin 1999)

"Iranul ca referinţă a lumii musulmane"

(une traduction plus littérale serait : "În Iran, Islamul luptă împotriva lui însuşi ».)

Dans l'exemple (1), le choix français est un titre percutant revêtant la forme d'une affirmation catégorique dans laquelle le sujet d'énonciation se trouve émotionnellement impliqué, tandis que, dans la version roumaine, l'information est exposée d'une façon plus neutre. Dans l'exemple (2), par contre, non seulement le titre roumain constitue une formulation nettement moins créative, mais il implique aussi une atténuation de l'émotion et de la tension véhiculées par le titre français.

Dans le deuxième grand groupe de mutations, observe Gemma Anjúdar Moreno, le titre français offre une information précise et idéologiquement marquée, qui est rendue en roumain d'une façon plus générale :

(3) - "Chantage nucléaire en Corée du Nord " (février 2003)

"O criză previzibilă şi evitabilă " (une traduction littérale : "Şantaj nuclear în Coreea de Nord ».)

Cet exemple partage la suppression dans la traduction de l'élément locatif ("Corée du Nord ") et la récupération de l'information par l'intermédiaire d'expressions plus générales. Dans la version en langue cible, le traducteur a proposé un titre moins précis qui, de par sa généralité, a plus de chances d'intéresser le lecteur roumain et de l'inciter à lire l'article. II s'agirait donc d'une autre façon de rendre le titre plus familier aux destinataires roumains. Il est évident que la version en langue cible de cette catégorie présente un éloignement sémantique très grand par rapport au titre français, mais on peut affirmer qu'elle partage aussi une caractéristique commune : les titres roumains sont moins percutants et créatifs qu'en français.

\section{Conclusions}

Les titres des articles journalistiques sont des segments courts et qui ont de multiples fonctions. Ils constituent donc un espace discursif complexe où se retrouvent des éléments de nature diverse (informatifs, idéologiques et culturels). Dans le cas du Monde diplomatique, les titres synthétisent d'une façon créative, percutante et idéologiquement marquée les positions qui seront argumentées ou réfutées par les auteurs des articles respectifs.

Gemma Anjúdar Moreno soutient que "le processus de traduction d'un texte met toujours en place une tension entre les structures linguistiques et discursives propres à la culture de départ et celle de la culture d'arrivée "(12). L'analyse contrastive que nous avons exposée ci-dessus a montré un échantillon varié de stratégies de traduction dans lesquelles le poids de

(12) Gemma Anjúdar Moreno, op. cit. 
la culture de départ est souligné pour orienter la démarche globale des traducteurs. On observe qu'en général le traducteur a choisi de respecter les particularités des titres français et que la traduction littérale constitue la modalité la plus fréquente et la moins perturbatrice du point de vue sémantique et pragmatique. Dans la traduction des titres de presse, c'est donc l'acceptabilité dans la culture journalistique d'arrivée qui cède la place aux considérations d'adéquation.

Cependant, on observe aussi que l'intervention du traducteur n'est pas homogène : on peut trouver des occurrences, moins fréquentes, qui montrent que l'acceptabilité a été mise au premier plan. Dans de tels cas, le traducteur a supprimé de façon presque systématique les marques propres au genre journalistique français pour obtenir un titre qui soit le plus naturel possible en fonction des conventions discursives de la culture d'arrivée. On observe que le traducteur peut pratiquer des manipulations diverses (modulations, modifications, mutations) pour " normaliser " la traduction des titres et les rendre adéquats dans la culture cible et conformes aux normes journalistiques de celle-ci. Selon Gemma Anjúdar Moreno, on pourrait placer ces occurrences aux niveaux les plus élevés de l'échelle de l'intervention du traducteur, car elles impliquent des modifications sémantiques et pragmatiques très importantes par rapport au titre original.

La question de l'adéquation et de l'acceptabilité du texte traduit confère une grande liberté de choix au traducteur : en fin de compte, il peut " manipuler " à son gré l'information contenue dans le titre de presse. Les choix de traduction répondent toujours à des motivations diverses et le traducteur peut accorder la priorité à l'adéquation au détriment de l'acceptabilité ou inversement. II ne faut pas oublier que chaque choix de traduction doit être motivé.

Pour résumer, nous devons souligner le fait que, lorsque les traducteurs mettent en place des stratégies non littérales, ils offrent des solutions inédites et font ressortir leur propre représentation cognitive du texte. Dans le même temps, ce sont les problèmes culturels qui surgissent en traduction : dans le cas de la presse écrite, ces divergences sont liées au genre discursif et à la traduction journalistique. La richesse et la variété des stratégies de traduction démontrent, une fois de plus, que la traduction est une rencontre et une tension entre deux cultures, deux visions du monde et deux traditions journalistiques différentes.

carmen.astirbei@gmail.com

Carmen-Ecaterina AŞTIRBEI est doctorante à l'université "Alexandru loan Cuza " de laşi, Roumanie et à l'Université de Bretagne-Sud de Lorient, France (thèse en cotutelle), sous la direction de Madame la professeur Marina Mureşanu et de Monsieur le professeur Jean Peeters. Son thème de recherche est "La poésie de Lucian Blaga et ses versions en français - figures de style et traduction ". Ses centres d'intérêt sont : la traductologie, la littérature et les études culturelles. Elle est l'auteure de plusieurs articles dans des revues telles que Loxias, Translationes, Acta lassyensia Comparationis, DICE (Diversité et identité culturelle en Europe), Atelier de Traduction. 


\section{Bibliographie}

ADAM, J.-M. (1990) : Éléments de linguistique textuelle. Théorie et pratique de l'analyse textuelle, Liège, Mardaga.

ANJÚDAR MORENO, Gemma, La traduction français-espagnol des titres journalistiques du Monde diplomatique, un exemple de tension entre adéquation et acceptabilité, http://www.translationdirectory.com/article1135.htm, consulté le 10 décembre 2010.

LEUVEN-ZWART, K. van (1989), Translation and Original: Similarities and Dissimilarities, Target 1 : 2, Amsterdam : John Benjamins.

MEERTENS, René, La traduction des textes journalistiques, http://www.foreignword.com, consulté le 10 décembre 2010.

NIDA, Eugene (2003), The Theory and Practice of Translation, Brill Academic Publishers. 